\title{
Gefitinib-coated balloon inhibits the excessive hyperplasia of intima after vascular injuries through PI3K/AKT pathway
}

\author{
Fen Tang ${ }^{\mathrm{a}, 1}$, Maojun Liu ${ }^{\mathrm{a}, 1}$, Ou Zeng ${ }^{\mathrm{a}}$, Wenting Tan ${ }^{\mathrm{a}}$, Junrong Long ${ }^{\mathrm{a}}$, Shengquan Liu $^{\mathrm{a}}$, \\ Jun Yang ${ }^{\mathrm{a}, *}$ and Chun $\mathrm{Chu}^{\mathrm{b}, *}$ \\ ${ }^{a}$ Department of Cardiology, the First Affiliated Hospital of University of South China, Hengyang, \\ Hunan, China \\ ${ }^{\mathrm{b}}$ Department of Pharmacy, the Second Affiliated Hospital of University of South China, Hengyang, \\ Hunan, China
}

\begin{abstract}
.
OBJECTIVE: To explore the effect of gefitinib-coated balloon suppressive action on the excessive hyperplasia of intima after balloon injury of common carotid artery in rats and on the PI3K/AKT signal pathway.

METHODS: MTT method and the expression of Bcl-2 and Caspase-3 proteins were detected in vitro; Adult SD rats were randomly split into 5 groups, namely sham group, model group, low-dosage gefitinib-coated balloon group, high-dosage gefitinibcoated balloon group, and paclitaxel-coated balloon group. The intimal proliferation of arteries, PCNA, P-AKT and PI3K protein expression, the cell apoptosis, expression of MMP9, TGF $\beta$ and IL6 mRNA were measured by hematoxylin and eosin (H\&E) staining, immunohistochemistry, TUNEL staining, and RT-qPCR.

RESULTS: At the same time and concentration, Gefitinib suppressed the proliferation of smooth muscle cell more significantly than paclitaxel. Bcl-2 and Caspase-3 in vascular smooth muscle and endothelial cells (VSMC, EC) were significantly downregulated and up-regulated after the cells were treated with gefitinib and paclitaxel. In gefitinib- and paclitaxel-coated balloon groups, significant up-regulations were found in the area of lumen. Furthermore, the expression of PCNA suggested that all coated balloons could suppress the excessive proliferation of smooth muscle cells in the hyperplastic intima compared with the control group. In gefitinib- and paclitaxel-coated balloon group, the expression of PI3K/AKT was significantly down-regulated. The use of drug-coated balloons mitigated the cell apoptosis in TUNEL. The expressions of MMP9, TGF $\beta$ and IL6 mRNA in the model group were obviously up-regulated; and they were obviously down-regulated in the high-dose gefitinib-coated balloon group compared with the model group.

CONCLUSIONS: Gefitinib-coated balloons were able to suppress the excessive proliferation in the common carotid arterial intima of rats more effectively than the paclitaxel-coated ones. The underlying mechanism may cover the PI3K/AKT signal pathway.
\end{abstract}

Keywords: Apoptosis, coated balloons, gefitinib, paclitaxel, proliferation, restenosis

\section{Introduction}

The migration and the proliferation of vascular smooth muscle cells (VSMCs) are the critical mecha-

\footnotetext{
${ }^{1}$ These authors contributed to the work equally and should be regarded as first co-authors.

${ }^{*}$ Corresponding authors: Yang Jun, Department of Cardiology, The First Affiliated Hospital of University of South China, 421001, No. 69 Chuanshan Road, ShiGu District, Hengyang, Hunan, China. Tel.: +1 18007340658; E-mail: yangjunincn@163.com; Chu Chun, Department of Pharmacy, The Second Affiliated Hospital of University of South China, 421001, No. 30 JieFang Road, ShiGu District, Hengyang, Hunan, China. Tel.: +86 13873409200; E-mail: yjchuchun @ 163.com.
}

0928-7329/19/\$35.00 (c) 2019 - IOS Press and the authors. All rights reserved

This article is published online with Open Access and distributed under the terms of the Creative Commons Attribution NonCommercial License (CC BY-NC 4.0). 
nisms, e.g. the restenosis after percutaneous coronary intervention (PCI). However, drug-coated balloon (DCB) can prevent the formation of restenosis effectively inside the nude stent by suppressing the intimal hyperplasia, which widens its application in vascular intervention. Rapamycin and paclitaxel are two types of non-selective cytotoxic drugs used for drug-coated stents and balloons for their ability to suppress the excessive proliferation of SMCs and mitigate the intimal repair [1]. Accordingly, cytotoxic drugs exhibiting more accurate selectivity on SMCs are the optimal choice for the new coating drugs.

Gefitinib, the $3^{\text {rd }}$ generation of antineoplastic drug, is a selective inhibitor on epidermal growth factor receptor tyrosine kinase (EGFR-TKIs). Proliferative SMCs and endothelial cells have been found to be able to express more EGFR than those in the resting state. Also, the preliminary studies revealed that gefitinib could selectively suppress SMCs' proliferation, and a selective cytotoxic effect in a dose-related degree was also observed in the endothelium and SMCs [2]. Thus, the expressions of Caspase- 3 and Bcl-2 apoptosis-related proteins were measured in-vitro experiments. Also, this study aimed to test the gefitinib-coated balloon performance in the in-vivo experiments to observe its suppressive effect on the excessive hyperplasia in intima of rats after their carotid common arteries were injured by balloons. Besides, the relevant mechanism of the intimal hyperplasia inhibition was investigated. Numerous studies showed that, PI3K-AKT signal pathway, a vital signal transduction pathway inside the cells, once being appropriately activated, can protect proliferation, migration and apoptosis of SMCs effectively $[3,4]$. PI3K/AKT signal way also serves as one of the critical downstream EGFR signal pathways, and EGFRTKIs inhibitor has been confirmed to be able to suppress the activation of PI3K-AKT signal pathway to regulate cell growth, proliferation and apoptosis according to the available research results [5-9]. For the P-AKT as the activation state of AKT, its measurement can reflect AKT's total amount [10]. TGF- $\beta$, a fibrosis-induced factor, can facilitate the accumulation of extracellular matrix components. Extracellular matrix is closely related with the proliferation and migration of smooth muscle cells. Matrix metalloproteinases (MMPs) are an enzyme family capable of degrading extracellular matrix, with a representative of MMP9. IL6, an important member of the interleukin family, will be expressed locally following vascular injury and generate pro-inflammatory effects. MMP9, TGF- $\beta$ and IL6 are involved in vascular remodeling mechanisms [11-13]. Some studies showed EGFR-TKIs inhibitor can suppress MMP9 and TGF- $\beta$ expression by suppressing PI3K-AKT signaling pathway activation to reduce the proliferation of smooth muscle cells $[14,15]$. This study aimed to investigate whether gefitinib-coated balloons could suppress cell proliferation or facilitate cell apoptosis by suppressing PI3K/AKT signal pathway activity and affect the intimal hyperplasia, which hinders the onset of restenosis after PCI.

\section{Basic experimental reagents and methods}

\subsection{Reagents and animals}

Hematoxylin, and TUNEL kit (Wellbio company, China). Chloral hydrate (Shanghai Shan Pu chemical company, China). Two-Step Kit (Golden Bridge Company, China). HRP Goat anti-rat IgG, BCL 2 rabbit polyclonal antibody (catalog numbers: 12789-1-AP, Proteintech Group, US). PCNA rabbit polyclonal antibody (catalog numbers: 10205-2-AP, Proteintech Group, US). Caspase-3 rabbit polyclonal antibody (catalog numbers: 19677-1-AP, Proteintech Group, US). Gefitinib and Taxol (Meilun biological company, China). The PTCA balloon catheter $(2.5 \mathrm{~F})$ and $1 \mathrm{~mm}$ balloon guide wire (Limited by Medtronic, Inc. US); P-AKT rabbit polyclonal antibody (SC: 514032, Ser 473, Santa Cruz Biotechnology, Inc, US), PI3K rabbit polyclonal antibody (SC: 293172,Santa Cruz Biotechnology, Inc, US); RT-Kit, Ultra SYBR Mixture and 100 bp DNA Marker (Com Win Biotech Co, Ltd, China). Rat aortic 
endothelial cells and smooth muscle cells (iCell, China). MTT kit (Beijing Saichi biotechnology company, China). DMEM medium culture fluid (Hyclone company, US). Fetal bovine serum and all the remaining reagents (Sigma, US). Fifty adult male Sprague-Dawley (SD) rats (weighing about 350 $400 \mathrm{~g}$ ) were provided by the Experimental Animal Center of South China University (China). All experimental protocols were approved by NanHua University's Review Committee for the Use of Animal Subjects.

\subsection{Cell cultures}

The newly purchased rat smooth muscle cells (Number: RAT-iCell-c004) and endothelial cells (Number: RAT-iCell-c003) were suspended in DMEM medium (containing 20\% fetal bovine serum, $100 \mathrm{IU} / \mathrm{ml}$ penicillin and $100 \mathrm{~g} / \mathrm{ml}$ streptomycin) and then cultured in an incubator containing $5 \% \mathrm{CO} 2$ at $37^{\circ} \mathrm{C}$.

\subsection{Cytotoxicity assays}

Bioassays were performed to determine the number of viable cells in proliferation or cytotoxicity tests by MTT, namely a colorimetric method. The MTT tetrazolium compounds were bio-reduced by cells into a colored formazan product, which is soluble in tissue culture medium and correlated with the cell number. Smooth muscle cells and endothelial cells were cultured using the above method and triturated into cell suspension $(3 \times 104 / \mathrm{ml})$ in the logarithmic growth phase, with 96 -well plate $200 \mu \mathrm{l} /$ well added, and then incubated in the incubator. Two $\mathrm{h}$ after cells adhered, paclitaxel $(1 \mu \mathrm{mol} / \mathrm{L})$ and gefitinib $(1 \mu \mathrm{mol} / \mathrm{L})$ were added separately for $48 \mathrm{~h}$ [2], incubated with $20 \mu \mathrm{l}$ MTT solution for $4 \mathrm{~h}$, and then adding $150 \mu \mathrm{l}$ SDS. The absorbance account was kept at $560 \mathrm{~nm}$ and, according to the measured values. The cell viability was calculated as the ratio of treated and control group optical densities (OD) multiplied by $100 \%$.

\subsection{Measurement of the expressions of Bcl-2 and Caspase-3 protein by western blotting}

One umol/L gefitinib and paclitaxel were used to treat VSMC and EC for $48 \mathrm{~h}$, respectively, another group of VSMC and EC no reagent added (control group), and total cell protein was extracted with RIPA kit. Bradford protein analysis kit was employed to measure the concentration of the extracted protein. The extracted protein was placed in EP tube and quantified by BCA colorimetry. After undertaking gel preparation (separation and concentration), comb placement, sample addition and electrophoresis, the protein was transferred to the PVDF membrane by the wet transfer method, and then sealed with the sealing liquid for $1 \mathrm{~h}$. The primary antibodies of GAPDH (dilution of 1:1000), Bcl-2, Caspase-3 (dilution of 1:500) were incubated at $37^{\circ} \mathrm{C}$ for $1 \mathrm{~h}$ and then incubated at $4^{\circ} \mathrm{C}$ in a refrigerator overnight, respectively. The membrane was washed three times with the newly prepared TBST, 10 min each time; the HRP labeled second antibodies (dilution of 1:8000) was incubated in a shaker at $37^{\circ} \mathrm{C}$ for $1 \mathrm{~h}$; After washing, developing and gray scanning, the expression levels of Bcl-2 and Caspase- 3 were expressed by the gray ratio of GAPDH internal reference band to the target band.

\subsection{Establishment of the animal model}

The experiment of this study strictly followed the rules of animal use and protection of the Experimen- 
tal Animal Research Institute of South China University (Hengyang, China). To ensure the diet access, a total of 50 male SD rats of weight from 300 to $330 \mathrm{~g}$ were placed into cages (1 rat/cage) for 1 week of feeding, in which the temperature was set as $24 \pm 3^{\circ} \mathrm{C}$, and the humidity of $60 \%$ was maintained. After being fully adapted to the environment, the rats were randomly split into 5 groups (10 rats in each group), namely sham group, control group, low-dosage gefitinib-coated balloon group $(10 \mathrm{mg} / \mathrm{kg})$, high-dosage gefitinib-coated balloon group $(30 \mathrm{mg} / \mathrm{kg})$ and paclitaxel-coated balloon group $(5 \mathrm{mg} / \mathrm{kg})$. Rats in the sham group underwent the separation of the right common carotid artery; the same procedure was performed for the rats of the control group, and the injuries were made using the ordinary balloon (equivalent to DMSO coated one), while balloons coated by gefitinib of different dosages or paclitaxel were used to cause injuries in rats from other groups. Procedures for injuring the rat common carotid artery using a balloon were as follows. Separation of right common carotid artery was performed in anesthetized rats; catheters of drug-coated or ordinary balloons were retrograded in a depth of $3.5 \mathrm{~cm}$ and connected to a hand-push pressure pump (USCI, US); under a pressure of $3 \mathrm{~atm}$, catheters were slowly withdrawn to the incision, and the pressure was then reduced to zero. After this step was repeated 3 times, the catheter was fully removed. Proximal segment was preserved for nearly 30 min using the vascular clamp. After 28 days after the operation, animals in each group were euthanized.

\subsection{Preparation of drug-coated balloons}

Gefitinib and paclitaxel were dissolved in DMSO to prepare the stock solutions ( $20 \mathrm{mmol} / \mathrm{L}$ ), which were then kept at $-20^{\circ} \mathrm{C}$. Before use, gefitinib and paclitaxel were diluted and evenly spread over the PTCA catheter balloons $(2.5 \mathrm{~F})$ following the animal weight and procedure. Next, balloons were dried for later use.

\subsection{Detecting the pathological changes in the common carotid artery via $H \& E$ staining}

After rats were euthanized, samples of their bilateral arteries were collected by being fixed in formaldehyde, dehydrated, cleared, embedded with paraffin and stained with hematoxylin-eosin following conventional methods. Balsam was dropped on the cleared section and sealed using cover glass. Subsequently, section was placed under the microscope $(100 \times)$ to be observed, and the images were analyzed using the Image-Pro-Plus software to calculate the area of intima. Samples collected from each rat were used to prepare 3 sections for consecutive measurements, and the average was taken as the result. With the tissues between the internal elastic membrane and surface of endothelium acting as the neointima, and with the tissues between the external and internal elastic membranes as the tunica intima, the areas of neointima and tunica intima could be directly measured using image analysis software.

\subsection{Detecting the protein expressions of PCNA, PI3K, and P-AKT through immunohistochemistry}

Dewaxed and rehydrated sections prepared as mentioned above underwent overnight incubation at $4^{\circ} \mathrm{C}$ with the use of the primary antibodies of PI3K, P-AKT and PCNA. Subsequently, the sections were washed and incubated with secondary antibodies (time, temperature?) and then underwent washing, $\mathrm{DAB}$ for peroxidase activity detection, washing, counterstaining, washing, bluing in ammonia water, dehydration and sealing. Sections were placed under the microscope for observation, and Image-Pro Plus 6.0 software was used to calculate the total optical density (OD) and staining area in the staining region. Next, the average of OD values was calculated. In each sample, the area of 5 visions under the microscope was measured, and the average was taken as the result. 
Table 1

Each primer sequence and product size of MMP9, TGF $\beta$ and IL6 mRNA

\begin{tabular}{clc}
\hline Gene & \multicolumn{1}{c}{ Primer sequence } & Product length/bp \\
\hline GAPDH & Forward ACAGCAACAGGGTGGTGGAC & 252 \\
& $\begin{array}{l}\text { Reverse TTTGAGGGTGCAGCGAACTT } \\
\text { MMP9 }\end{array}$ & $\begin{array}{l}\text { Forward CCCCGAGACCTGAAAACCTCCAAC } \\
\text { Reverse GGCCTTTAGTGTCTCGCTGTCCA }\end{array}$ \\
TGF $\beta 1$ & $\begin{array}{l}\text { Forward CCGCTTCTGCTCCCACTCCC } \\
\text { Reverse CGTTTCACCAGCTCCATGTCGAT }\end{array}$ & 189 \\
IL6 & $\begin{array}{l}\text { Forward TCACTATGAGGTCTACTCGG } \\
\text { Reverse CATATTGCCAGTTCTTCGTA }\end{array}$ & 141 \\
\hline
\end{tabular}

\subsection{Detecting cell apoptosis in the hyperplastic intima via TUNEL staining}

TUNEL staining was performed on dewaxed section samples accordingly with the manufacturer's instructions. Subsequently, sections were sealed and placed under the microscope to be observed. Images were loaded to the Image-Pro Plus software to be analyzed. For each sample, 5 visions under the microscope were used to calculate the positive expression rate, and the average was taken as the result. The positive rate was equal to the quantity of cell nuclei with positive expression under a single vision divided by the total number of cell nuclei under this vision.

\subsection{Determining the relative expression of MMP9, TGF $\beta$ and IL6 $m R N A$ by RT-qPCR method}

Total RNA was extracted from rat vascular tissue and then served as the template for cDNA reverse transcription. The mentioned operations were performed strictly following the instructions. RT-qPCR quantification was performed by SYBR Green method. Each primer sequence and product size are listed in Table 1. Gel electrophoresis was performed for the amplification products, and photos were captured. The solubility curve suggested that the PCR reaction product was separate double-stranded DNA. With GAPDH as the internal reference, the relative expressions of MMP9, TGF $\beta$ and IL6 mRNA were determined using $\Delta \Delta \mathrm{Ct}$ value method.

\subsection{Statistical analysis}

Statistical analysis of the mentioned data was conducted using the SPSS 18.0 statistical software package. Measurement data are denoted as mean \pm standard deviation, and statistical differences between the groups were assessed by one-way analysis of variance (ANOVA). The calculated value $P<0.05$ suggested that the difference was statistically significant.

\section{Results}

\subsection{Cytotoxic effects of gefitinib on smooth muscle cells and endothelial cells}

Figure 1 shows that the proliferation of endothelial and smooth muscle cells was significantly suppressed by gefitinib and paclitaxel, whereas at the same time and the same concentration $(P<0.05)$ gefitinib exhibited stronger suppressive effect on the proliferation of smooth muscle cells and of endothelial cells than paclitaxel, which is consistent with the results achieved previously [2]. 


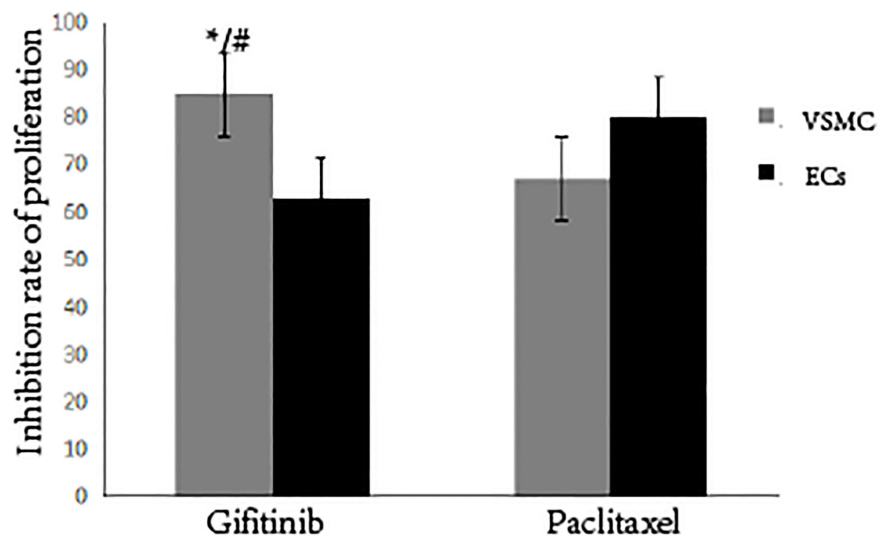

Fig. 1. Comparison of gifitinib and paclitaxel effect on inhibition rate of cell proliferation. Note: As compared to paclitaxel inhibition to VSMC, ${ }^{*} P<0.05$. As compared to gefitinib inhibition to ECs, ${ }^{\#} P<0.05$.

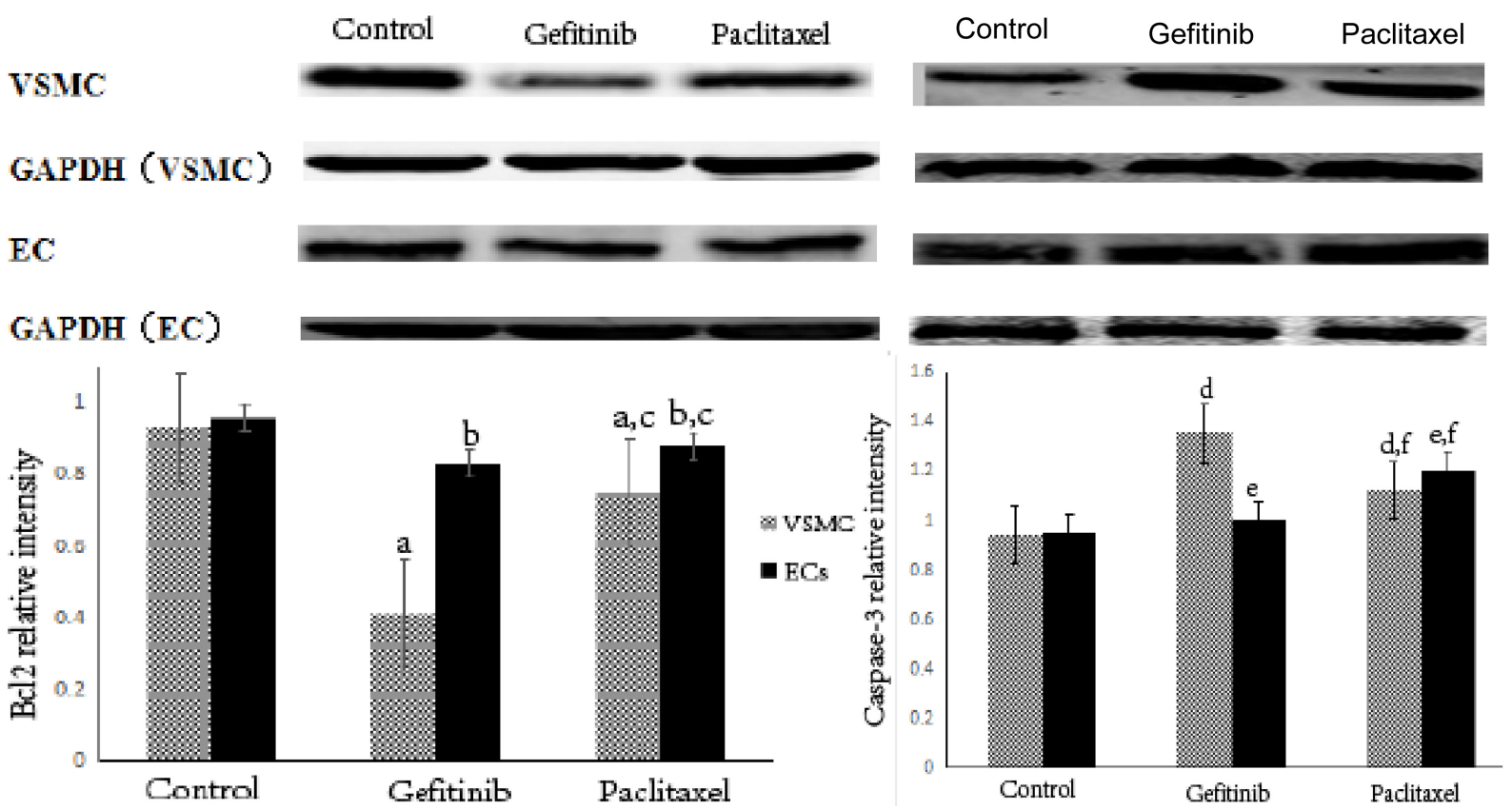

Fig. 2. The expression of Bcl2 and Caspase-3 in different cells. Note: Compare with control group, ${ }^{\text {a,b,d,e }} P<0.05$, Comparison with gefitinib groups, ${ }^{\mathrm{c}, \mathrm{f}} \mathrm{B}<0.05$.

\subsection{Expression of Bcl-2 and Caspase-3 proteins after being treated with two types of cell drugs}

As shown in the Fig. 2, for VSMC, the expression of Bcl-2 protein in the gefitinib group and the paclitaxel group was significantly down-regulated, while the expression of Caspase-3 protein was significantly up-regulated compared with the Control group $(P<0.05)$; for EC, Bcl-2 protein's expression was significantly down-regulated, and Caspase-3 protein's expression was significantly up-regulated in the gefitinib group and paclitaxel group compared with the Control group $(P<0.05)$, whereas VSMC varied more significantly; under the trestment of the two drugs, the gray ratios of Bcl-2 and Caspase- 3 

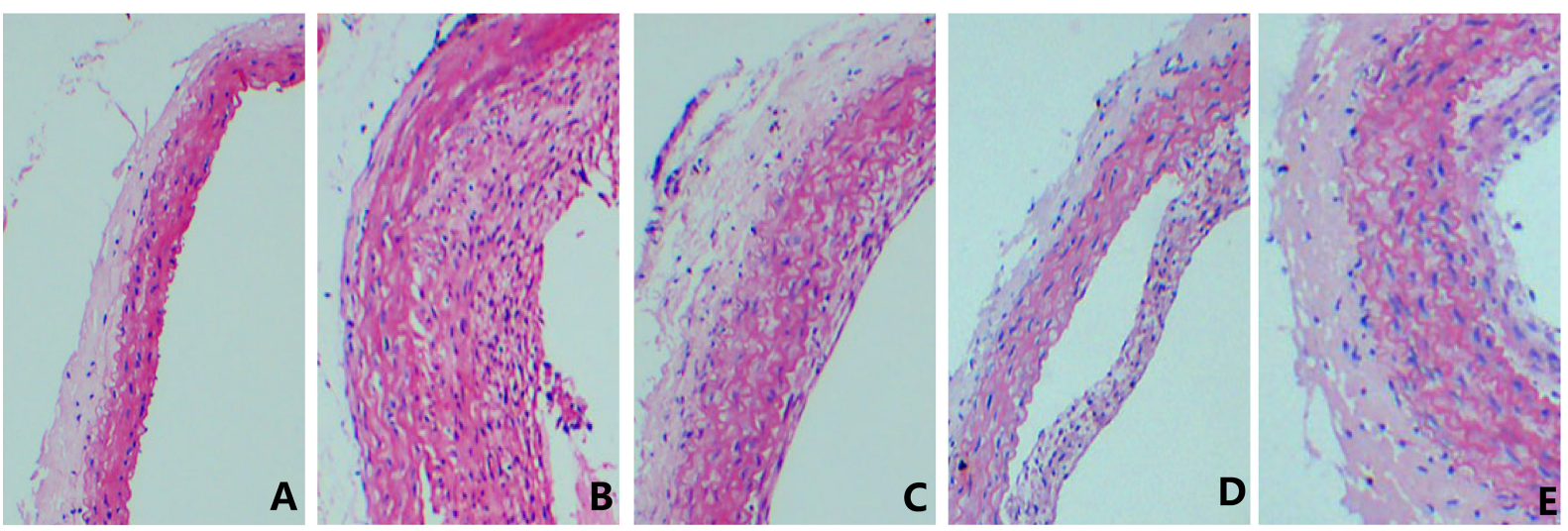

Fig. 3. The influence on vascular lumen area. Note: A: Sham operation group; B: Model group; C: Low-dosage gefitinib group; D: High-dosage gefitinib group; E: Paclitaxel group.

in the paclitaxel group were significantly up-regulated $(P<0.01)$ compared with those in the gefitinib group $(P<0.01)$.

\subsection{Detecting the pathological changes in common carotid artery via H\&E staining}

Figure 3 suggests that a significant increase in the thickness of common carotid artery and obvious stenosis in lumen were identified in the control group rats compared with the sham operation group; compared with the model group, alleviations were found in the hyperplasia of intima in common carotid arteries of the rats in the low- and high-dosage gefitinib-coated balloon groups and the paclitaxel-coated balloon group, and stenosis in lumen was also significantly improved, especially in the high-dosage gefitinib-coated balloon group. However, no statistically significant difference was found in comparison of intimal hyperplasia between the low-dosage gefitinib- and the paclitaxel-coated balloon groups.

\subsection{Detecting the protein expression of PCNA, P-AKT, and PI3K via immunohistochemistry}

Figure 4 shows that compared with the sham operation group, the protein expression of PCNA, PAKT, and PI3K in the hyperplastic intima in common carotid arteries of the model group rats was significantly augmented $(P<0.01)$. Compared with the model group, PCNA, P-AKT, and PI3K were down-regulated in the hyperplastic intima in common carotid arteries of the rats of the paclitaxel-, low-, and high-dosage gefitinib-coated balloon groups, whereas stenosis in lumen was also significantly improved $(P<0.05)$, especially in the high-dosage gefitinib-coated balloon group. Besides, the protein expressions of PCNA, P-AKT, and PI3K were significantly lower than those in the paclitaxel-coated balloon group $(P<0.01)$. However, in the low-dosage gefitinib-coated balloon group, only protein expression of P-AKT was down-regulated $(P<0.01)$, and no statistically significant difference was found by comparing the protein expression of PCNA and PI3K for the two groups $(P>0.05)$.

\subsection{Detecting cell apoptosis in the hyperplastic intima via TUNEL staining}

Figure 5 indicates a significant increase in the apoptotic cells in hyperplastic intima of common carotid artery of the model group rats, compared with the sham operation group ones $(P<0.01)$. Compared with the model group, dramatic decrease of the apoptotic cells was revealed in the paclitaxel-, low-, 

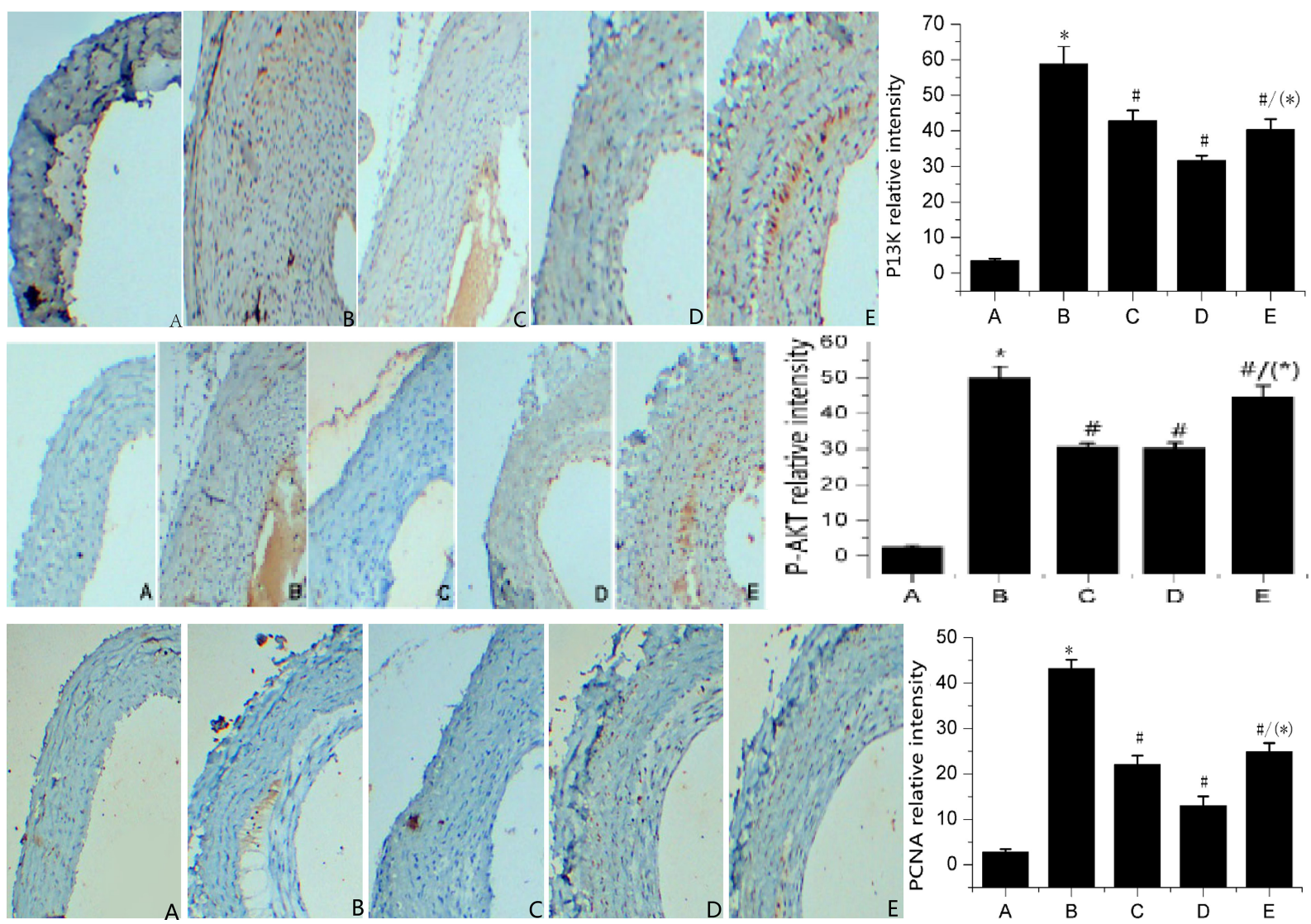

Fig. 4. Protein expressions of PI3K, P-AKT, and PCNA for investigated groups. Note: A: Sham operation group; B: Model group; C: Low-dosage gefitinib group; D: High-dosage gefitinib group; E: Paclitaxel group; Comparison with sham operation group yields ${ }^{*} P<0.01$; Comparison with the model group yields ${ }^{\#} P<0.05$; Comparison with the high- and low-dosage gefitinib groups yields ${ }^{(*)} P<0.01$ and ${ }^{(\#)} P>0.05$, respectively.

and high-dosage gefitinib-coated balloon groups, whereas the stenosis in lumen was also significantly improved $(P<0.05)$. The above reductions were the most significant in the high-dosage gefitinib- and paclitaxel-coated balloon groups. However, no statistically significant difference was reported between the low-dosage gefitinib-coated balloon group and the paclitaxel-coated one $(P>0.05)$.

\subsection{Determining the relative expression of MMP9, TGF $\beta$ and IL6 $\mathrm{mRNA}$ by RT-qPCR method}

Figure 6 suggests that compared with the sham operation group, the expression of MMP9, TGF $\beta$ and IL6 mRNA in the hyperplastic intima of rat's common carotid artery in the model group were obviously up-regulated $(P<0.05)$; compared with the model group, MMP9, TGF $\beta$ mRNA in the hyperplastic intima of rat's common carotid artery were obviously down-regulated in the high-dose gefitinib-coated balloon group and the paclitaxel-coated balloon group $(P<0.05)$, and IL6 in the high-dose gefitinibcoated balloon group was also down-regulated $(P<0.05)$ but not in the paclitaxel-coated balloon $\operatorname{group}(P>0.05)$; no statistically significant difference was reported between the high-dosage gefitinibcoated balloon group and the paclitaxel-coated one $(P>0.05)$. 

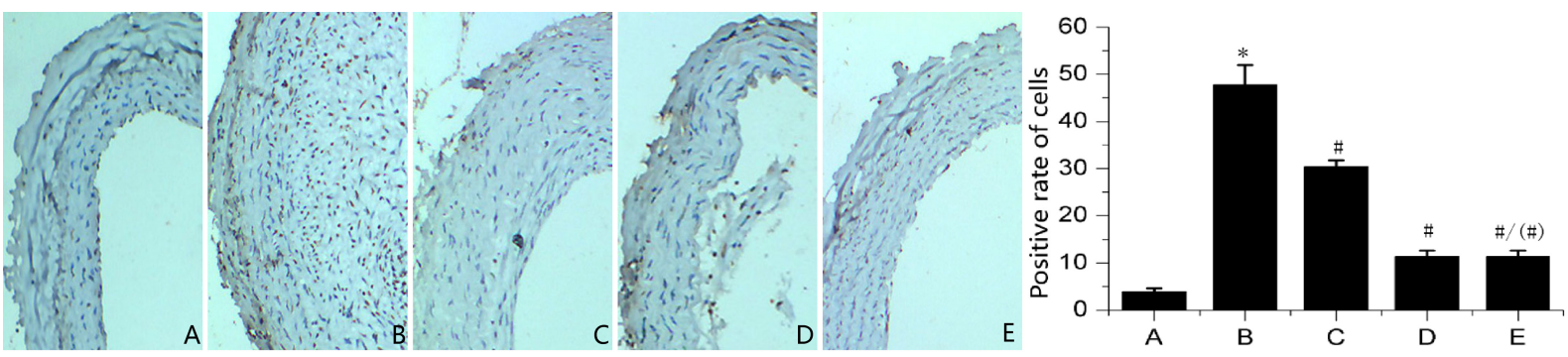

Fig. 5. Growth of apoptotic cells in the investigated groups. Note: A: Sham operation group; B: Model group; C: Low-dosage gefitinib group; D: High-dosage gefitinib group; E: Paclitaxel group; Comparison with sham operation group yields ${ }^{*} P<$ 0.01 ; Comparison with the model group yields ${ }^{\#} P<0.05$; Comparison with the high- and low-dosage gefitinib groups yields ${ }^{(\#)} P>0.05$.
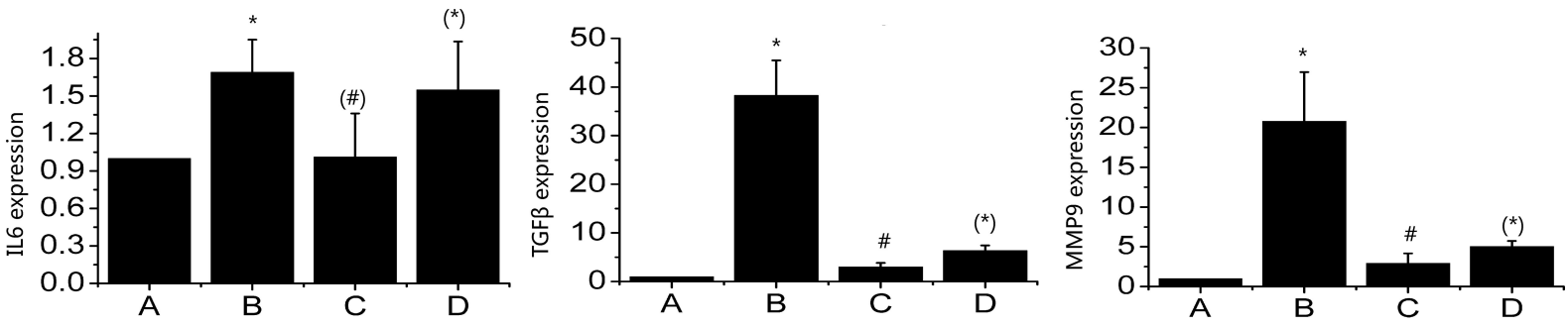

Fig. 6. Expression levels of MMP9, TGF $\beta$ and IL6 in hyperplastic intima tissues from each group groups. Note: A: Sham operation group; B: Model group; C: High-dosage gefitinib group; D: Paclitaxel group; Comparison with sham operation group yields ${ }^{*} P<0.05$; Comparison with the model group yields ${ }^{\#} P<0.05$; ${ }^{\left({ }^{*}\right)} P>0.05$, Comparison with the high-dosage gefitinib groups yields ${ }^{(*)} P>0.05$.

\section{Discussion}

Percutaneous transluminal coronary angioplasty (PTCA) has become one of the regular vascular intervention treatment methods, yet the incidence rate of restenosis in 6 months after implantation of nude stent, reaching 10 to $20 \%$, makes it the basic postoperative complication after intervention therapy [16]. The pathogenesis of restenosis after stent implantation is primarily correlated with the migration of VSMCs to intima and the continuous proliferation of VSMCs. At present, to reduce the incidence rate of stenosis after PCI, drug-coated stents (DCS) and DCB have been widely applied clinically, and the latter gained various achievements in prophylaxis and treatment of restenosis. Numerous studies reported that DCB, a novel instrument used for the intervention treatment for coronary heart disease, have exhibited promising safety and high effectiveness in prophylaxis of restenosis [17-19]. The major principle of DCB is that the drugs used for their coating should be rapidly released and absorbed by vascular wall, thereby rapidly suppressing the excessive proliferation of intima after vascular injuries [20]. However, paclitaxel or rapamycin, used as the drugs of DCS or DCB coatings, not only suppresses the excessive proliferation of intima after vascular injuries, but also hinders the migration and repair of endothelial cells, thereby severely affecting the endothelialization of stent after PCI and inducing the in-stent thrombosis or restenosis. Thus, cytotoxic drugs with a more accurate selectivity on SMCs will be the best choice for new generation of coating drugs.

Gefitinib is a selective inhibitor on EGFR-TKIs. Some researchers reported that proliferative SMCs and endothelial cells can express more EGFR than those in the resting state, In the in-vitro experiment 
of this study, we also found that gefitinib inhibits the proliferation of SMCs more effectively than paclitaxel but exhibits a relatively weak cytotoxic effect on endothelial cells, with a selective cytotoxic effect being also revealed in the endothelium and SMCs. Accordingly, the results achieved strongly suggest that gefitinib, a DCB coating drug, can effectively suppress the excessive SMC proliferation and intimal hyperplasia, while reducing the injuries to endothelial cells. However, the available in-vivo experimental results are insufficient to clarify whether gefitinib can effectively suppress the excessive SMC proliferation and intimal hyperplasia. In this study, gefitinib-coated DCB were used to observe the suppressive effect on excessive hyperplasia of intima after the common carotid artery of rats was injured by these DCB. The results showed that high- or low-dosage gefitinib-coated balloons can sufficiently suppress the excessive hyperplasia of intima after the common carotid artery of rats was injured with significant alleviations in stenosis of lumen in contrast to the sham group. Compared with the paclitaxel-coated DCB, high-dosage gefitinib-coated ones exhibit a stronger suppressive effect on the excessive proliferation of intima. These in-vivo experimental results revealed that gefitinib, a DCB coating drug, can effectively suppress the excessive hyperplasia of intima after the common carotid artery of rats was injured, and, thus, it can be recommended as a new-generation coating drug, while gefitinib-coated balloons, with a promising application, can be used in the new techniques for prophylaxis and treatment of restenosis after PCI.

Gefitinib, a member of EGFR-TKI family, is capable of regulating cell proliferation, differentiation and apoptosis by modulating the activation of the EGFR signal pathway [21]. The PI3K-AKT signal pathway is a vital part of the EGFR downstream signal pathway, and PI3K-AKT signal pathway plays critical roles in regulating the cell cycle, cell growth, proliferation and apoptosis according to previous studies [22-24]. PCNA, as an auxiliary protein of DNA polymerase, participates in the DNA synthesis and serves as a specific indicator of cell proliferation [25]. In this study, it has been found that, compared with the sham group, PCNA protein expression was significantly increased in the hyperplastic intima in the common carotid artery of rats in the control group, suggesting that rapid proliferation occurs in SMCs in the hyperplastic intima after intimal injuries. However, compared with the control group, dramatic decreases were found in the protein expressions of PCNA in the hyperplastic intima of the rats in the low- and high-dosage gefitinib-coated balloon groups and the paclitaxel-coated balloon group, indicating that gefitinib- and paclitaxel-coated balloons can suppress the proliferation of SMCs in the hyperplastic intima, whereas the suppressive effect in the high-dosage group is more obvious. Moreover, apoptosis is vital for the occurrence and development of many cardiovascular diseases. When the body is long subject to the action of various pro-apoptotic factors, Caspases cascade pathways in tissues can be activated in sequence, thereby inducing apoptosis. Bcl-2 has been proved to block the activation of caspase and suppress apoptosis [26]. Western blot results of the in vitro experiment here showed that the expression of $\mathrm{Bcl}-2$ protein of the two groups was and the expression of caspase- 3 protein were significantly down-regulated and significantly up-regulated, respectively, after 1umol/L gefitinib and paclitaxel were applied to VSMC and EC for $48 \mathrm{~h}$, respectively. For the two types of cells, the variation of gray ratio was more obvious in the gefitinib group. This further revealed that gefitinib and paclitaxel had suppressive effects on both VSMC and EC and both promoted apoptosis. However, gefitinib had significantly stronger suppressive effects on VSMC than paclitaxel, while its inhibitory effects on EC were weaker than those of paclitaxel. Accordingly, it is further proved that gefitinib has a strong suppressive effect on VSMC and a weak suppressive effect on EC. In this experiment, the variations of apoptosis in hyperplastic endometrium were also observed in vivo. The results showed that apoptosis was found in the hyperplastic endometrium of the model group. This result is also similar to our in vitro experiment results and the results observed by other scholars, which may be related to the rapid 
increase of cell density caused by the excessive proliferation of smooth muscle cells in the hyperplastic endometrium, thereby suppressing cell contact and the induced apoptosis. It has been reported that gefitinib can suppress the PI3K-AKT signaling pathway by regulating the phosphorylation of mammalian target of rapamycin (mTOR) [27]. In this study, P-AKT and PI3K protein expressions were significantly up-regulated in the intima of carotid artery hyperplasia in the model group compared with those of the sham group. However, P-AKT's and PI3K's expressions were significantly down-regulated in the hyperplasia intima of rats in the low and high dose gefitinib balloon group and paclitaxel balloon group, which is consistent with the PI3K-AKT signaling pathway expression that we have previously studied in vitro. The results suggested that the mechanism of gefitinib and paclitaxel coated balloon suppressing endometrial hyperplasia was primarily associated with the suppression of smooth muscle cell hyperplasia and the promotion of apoptosis, and the specific suppressing mechanism of smooth muscle cell proliferation may have a relation to the suppression of the PI3K/AKT pathway. However, in-vivo experiments suggested that the proliferation of smooth muscle cells in the hyperplastic endometrium was suppressed after drug balloon intervention, and the cell contact suppression and apoptosis induced by it were significantly reduced. Thus, the primary mechanism is still considered to inhibit cell proliferation. TGF- $\beta$ is one of the vital fibrogenic cytokines, robust in promoting extracellular matrix deposition. Studies have shown that the extracellular matrix is closely related to the proliferation and migration of smooth muscle cells. Matrix metalloproteinases (MMPs) are a family of enzymes that can degrade the extracellular matrix, and MMP9 is one of its representatives [28]. When studying the effect of valsartan, some researchers found that MMP9 is involved in the proliferation and migration of smooth muscle [29]. As a critical member of the Interleukin family, Interleukin 6 (IL6) can be highly expressed by injured vascular smooth muscle cells, thereby exerting a proinflammatory effect [13]. Clinical studies have also reported that IL-6 mRNA is highly expressed in atherosclerotic plaques. In this study, total RNA was extracted from rat blood vessels, and the expressions of MMP9, TGF- $\beta$ and IL6 genes were determined by RT-qPCR. The results suggested that compared with the sham group, MMP9, TGF- $\beta$ and IL6 gene expressions were significantly up-regulated in the intima of carotid artery hyperplasia in the model group. Compared with the model group, the expression of MMP9, TGF- $\beta$, and IL6 genes in the intima of common carotid artery hyperplasia in the rats were significantly down-regulated in the high-dose gefitinib balloon group and paclitaxel balloon group. In the meantime, compared with the paclitaxel group, the reduction was more obvious in the high-dose gefitinib balloon group. Gefitinib can inhibit the expression of MMP9, TGF- $\beta$, etc., thereby reducing the proliferation of smooth muscle cells, and its effect is stronger than paclitaxel. Existing studies revealed that EGFR-TKIs inhibitors can reduce the proliferation of smooth muscle cells by suppressing the activation of the PI3K/AKT signaling pathway and inhibiting the expression of MMP9 and TGF- $\beta$, etc. [15-17]. Accordingly, the authors speculated that the mechanism of action here may be associated with the suppression of the activation of the PI3K-AKT signaling pathway.

This study helps us understanding the suppressive effect of gefitinib on VSMC proliferation and its possible mechanism and provides a theoretical basis for the screening of stent-coated drugs in patients with coronary heart disease.

\section{Conclusion}

The results of this study prove that gefitinib-coated balloons suppress the excessive proliferation of SMC through regulating the PI3K-AKT signal pathway to block the intimal hyperplasia after injuries caused by balloon with a similar effect to the paclitaxel-coated balloons. However, the expression of the 
PI3K/AKT pathway in-vivo which detected by western blotting method requires further experimentation. Furthermore, endothelial protection and more effective safety evaluation need further studies, and this is also our next research direction.

\section{Acknowledgments}

The authors gratefully acknowledge the financial supports of the National Natural Science Foundation of China (Grant nos. 81270181 and 1202830).

\section{Conflict of interest}

None to report.

\section{References}

[1] O'Donnell NG, Asbury AJ. Sirolimus- and taxol-eluting stents differ towards intimal hyperplasia and reendothelialization. Journal of Invasive Cardiology, 2005, 17: 497-502.

[2] Zhixiong W, Jian L, Fang L, et al. Gefitinib Can More Specifically Inhibit Smooth Muscle Cell Proliferation Compared with Paclitaxel as A New Stent Coating Material. In: Electrical and Control Engineering \& Materials Science and Manufacturing: The Proceedings of Joint Conferences of the 6th (ICECE2015) and the 4th (ICMSM2015).

[3] Vahidnezhad H, Youssefian L, Uitto J. Molecular genetics of the PI3K-AKT-mTOR pathway in genodermatoses: diagnostic implications and treatment opportunities. J Investigative Dermatology, 2015, 136(1): 15-23.

[4] Xia P, Xu XY. PI3K/Akt/mTOR signaling pathway in cancer stem cells: from basic research to clinical application. American Journal of Cancer Research, 2015, 5: 1602-1609.

[5] Wu H, Fan F, Liu Z, Shen C, Wang A, Lu Y. Norcantharidin combined with EGFR-TKIs overcomes HGF-induced resistance to EGFR-TKIs in EGFR mutant lung cancer cells via inhibition of Met/PI3k/Akt pathway. Cancer Chemotherapy and Pharmacology, 2015, 76: 307-315.

[6] Yip PY. Phosphatidylinositol 3-kinase-AKT-mammalian target of rapamycin (PI3K-Akt-mTOR) signaling pathway in non-small cell lung cancer. Translationallung Cancer Research, 2015, 4: 165-176.

[7] Kim GD. Hesperetin inhibits vascular formation by suppressing of the PI3K/AKT, ERK, and p38 MAPK signaling pathways. Preventive Nutrition and Food Science, 2014, 19: 299-306.

[8] Li Y, Fu S, Chen H, Feng Q, Gao Y, Xue H. Inhibition of endothelial Slit2/Robo1 signaling by thalidomide restrains angiogenesis by blocking the PI3K/Akt pathway. Digestive Diseases and Sciences, 2014, 59: 2958-2966.

[9] Tetsu O, Phuchareon J, Eisele DW, Hangauer MJ, Mccormick F. AKT inactivation causes persistent drug tolerance to EGFR inhibitors. Pharmacological Research, 2015, 102: 132-137.

[10] Kunter I, Erdal E, Nart D, Yilmaz F, Karademir S, Sagol O. Active form of AKT controls cell proliferation and response to apoptosis in hepatocellular carcinoma. Oncology Reports, 2014, 31: 573-80.

[11] Segarra M, Garcíamartínez A, Snchez M, Hernndezrodríguez J, Lozano E, Grau JM. Gelatinase expression and proteolytic activity in giant-cell arteritis. Annals of the Rheumatic Diseases, 2007, 66: 1429-35.

[12] Zabini D, Granton E, Hu Y, Miranda MZ, Weichelt U, Breuils BS. Loss of SMAD3 promotes vascular remodeling in pulmonary arterial hypertension via MRTF disinhibition. American Journal of Respiratory \& Critical Care Medicine, 2017, 1.

[13] Savale L, Tu L, Rideau D, Izziki M, Maitre B, Adnot S. Impact of interleukin-6 on hypoxia-induced pulmonary hypertension and lung inflammation in mice. Respiratory Research, 2009, 10: 6.

[14] Normanno N, Gullick WJ. Epidermal growth factor receptor tyrosine kinase inhibitors and bone metastases: different mechanisms of action for a novel therapeutic application? Endocrine-related Cancer, 2006, 13: 3.

[15] Liu Y, Wang Z, Kwong SQ, Lui EL, Friedman SL, Li FR. Inhibition of PDGF, TGF- $\beta$, and Abl signaling and reduction of liver fibrosis by the small molecule Bcr-Abl tyrosine kinase antagonist Nilotinib. Journal of Hepatology, 2011, 55: 612.

[16] Meraj PM, Jauhar R, Singh A. Bare metal stents versus drug eluting stents: where do we stand in 2015. Curr Treat Options Cardiovasc Med, 2015, 17: 393. 
[17] Scheller B, Speck U, Abramjuk C, Bernhardt U, Böhm M, Nickenig G. Paclitaxel balloon coating, a novel method for prevention and therapy of restenosis. Circulation, 2004, 110: 810-814.

[18] Xu B, Gao R, Wang J, Yang Y, Chen S, Liu B. A prospective, multicenter, randomized trial of paclitaxel-coated balloon versus paclitaxel-eluting stent for the treatment of drug-eluting stent in-stent restenosis: results from the PEPCAD China ISR trial. JACC Cardiovasc Interv, 2014, 7: 204-211.

[19] Gao L, Chen YD. Application of drug-coated balloon in coronary artery intervention: Challenges and opportunities. J Geriatr Cardiol, 2016, 13: 906-913.

[20] Cortese B, Bertoletti A. Paclitaxel coated balloons for coronary artery interventions: a comprehensive review of preclinical and clinical data. Int J Cardiol, 2012, 161: 4-12.

[21] Herbst RS. Review of epidermal growth factor receptor biology. Int J Radiat Oncol Biol Phys, 2004, 59: 21-26.

[22] Li L, Wang S, Zheng F. Chinese herbal medicine Fuzheng Kang-Ai decoction sensitized the effect of gefitinib on inhibition of human lung cancer cells through inactivating PI3-K/Akt -mediated suppressing MUC1 expression. Ethnopharmacol, 2016, 194: 918-929.

[23] Zhou G, Zhang F, Guo Y, Huang J, Xie Y, Yue S. MiR-200c enhances sensitivity of drug-resistant non-small cell lung cancer to gefitinib by suppression of PI3K/Akt signaling pathway and inhibits cell migration via targeting ZEB1. Biomed Pharmacother, 2017, 85: 113-119.

[24] Gadgeel SM, Wozniak A. Preclinical rationale for PI3K/Akt/mTOR pathway inhibitors as therapy for epidermal growth factor receptor inhibitor-resistant non-small-cell lung cancer. Clin Lung Cancer, 2013, 14: 322-32.

[25] Takada M, Tanaka H, Yamada T, Ito O, Kogushi M, Yanagimachi M. Antibody to thrombin receptor inhibits neointimal smooth muscle cell accumulation without causing inhibition of platelet aggregation or altering hemostatic parameters after angioplasty in rat. Circ Res, 1998, 82: 980-987.

[26] Quinn L. Buffy, a Drosophila Bcl-2 protein, has anti-apoptotic and cell cycle inhibitory functions. EMBO (European Molecular Biology Organization) Journal, 2003, 22(14): 3568-3579.

[27] Moore JC, Sumerel JL, Schnackenberg BJ, Nichols JA, Wikramanayake A, Wessel GM. Cyclin D and cdk4 are required for normal development beyond the blastula stage in sea urchin embryos. Mol Cell Biol, 2002, 22: 4863-75.

[28] Hasegawa A, Sato K, Shirai R, Watanabe R, Yamamoto K, Watanabe K. Vasoprotective effects of urocortin 1 against atherosclerosis in vitro and in vivo. Plos One, 2014, 9: e110866.

[29] Yao JS, Chen Y, Zhai W, Xu K, Young WL, Yang GY. Minocycline exerts multiple inhibitory effects on vascular endothelial growth factor-induced smooth muscle cell migration the role of ERK1/2, PI3K, and matrix metalloproteinases. Circulation Research, 2004, 95: 364-71. 